\title{
An informationally complete Wigner function for the Tavis-Cummings model \\ Visualization of cat swapping and quantum correlations in field-many atom systems
}

\author{
Russell P Rundle · Mark J Everitt
}

Received: date / Accepted: date

\begin{abstract}
Here we consider an informationally complete Wigner function approach to look at multiple atoms (qubits) coupled to a field mode. We consider the Tavis-Cummings interaction between a single field mode with two qubits and then with five.
\end{abstract}

Keywords Hybrid Wigner function · Light-Matter interaction · Tavis-Cummings Model · Phase space

\section{Introduction}

The Jaynes-Cummings model describes a simplified model of an atom coupled to an electromagnetic field in the rotating wave approximation 1]. It takes the form of a harmonic oscillator - for the field mode - coupled to a two level system - for two states of an atom. One of the crucial features of this model is that the system realizes a process where a field mode prepared in a coherent state will evolve into a Schrödinger cat state; that is to good approximation a separable state. This makes the setup appealing for continuous mode quantum information processing applications.

If one introduces more atoms coupled to the same field in the same way then the behaviour can get even more interesting, this is known as the Tavis-Cummings model 2], where in the interaction picture the Tavis-Cummings model is described

R. P. Rundle

School of Mathematics, Fry Building, University of Bristol, United Kingdom

Wolfson School of Mechanical, Electrical and Manufacturing Engineering, Loughborough University, Leicestershire, United Kingdom

Quantum Systems Engineering Research Group, Department of Physics, Loughborough University, Leicestershire, United Kingdom

E-mail: r.rundle@bristol.ac.uk

\section{J. Everitt}

Quantum Systems Engineering Research Group, Department of Physics, Loughborough University, Leicestershire, United Kingdom

E-mail: mark.j.everitt@physics.org 
by the Hamiltonian

$$
\hat{H}_{\mathrm{TC}}=\sum_{i}^{N}\left(\hat{a}^{\dagger} \hat{\sigma}_{-}^{(i)}+\hat{a} \hat{\sigma}_{+}^{(i)}\right),
$$

where $\hat{\sigma}_{ \pm}^{(i)}$ is the operator $\hat{\sigma}_{ \pm}=\left(\hat{\sigma}_{x} \pm \mathrm{i} \hat{\sigma}_{y}\right) / 2$ that acts on the $i^{\text {th }}$ atom, and $\hat{a}\left(\hat{a}^{\dagger}\right)$ is the standard annihilation (creation) operator for the field mode.

Starting the multi-qubit system in an atomic Schrödinger cat state and the field mode in a coherent state, one finds that the Schrödinger cat state will swap between the atoms and the field mode. This behaviour we will refer to as collapse and revival of the Schrödinger cat state. Once more this has potential for continuous mode quantum information processing applications.

For one atom and a field mode initially prepared in a coherent state with an average of $\bar{n}$ photons, and an atom field coupling strength of $g$, there are three timescales that are important. The first is the Rabi time $t_{R}=\pi /(g \sqrt{\bar{n}})$; the next sets the envelope of the atomic inversion $t_{c}=\sqrt{2} / g$, which is a Gaussian envelope and in this way distinguishable from damping effects; the last and most important is the first revival time $t_{r}=2 \pi \sqrt{\bar{n}} / g$. As we increase the number, $N$, some of these time scales change [1, 3]. The most important is that the first revival time is $t_{r_{1}}=t_{r} / N$.

This scaling led to the idea that introducing more atoms to a system could in fact be a mechanism for overcoming decoherence for some continuous mode quantum information processing applications providing the dominating damping terms were in the field mode [4. This study made use of a Wigner function of a general angular-momentum state as well as the usual Wigner function for the field mode to visualise the various effects of decoherence on the system's dynamics 5. Importantly the Wigner function of the atomic system could be used to demonstrate that, as the number of qubits increased, the effect decoherence on the cat-swap process is reduced. We note here that that that result also indicates that by measuring negative values this could lead to an efficient verification and validation procedure.

The issue with mapping this system's state onto an angular momentum state is that it is not necessarily bijective, where it only allows the representation of states that are symmetric under permutation of atoms. They only map atomic states by a Dicke mapping of a single given value of angular momentum 6 . For the study of a cat-swap process, this was not an issue as both the Tavis-Cummings Hamiltonian as field decoherence conserved angular momentum. This is not generally true for more realistic system Hamiltonians or environments.

What we describe in this paper is the complete representation in phase space for systems such as those described by the Tavis-Cummings model. We illustrate how signatures of certain important quantum correlation, or their absence, manifest. One of the key observations is that, for pure states, signatures of entanglement can be clearly seen if certain visualisation methods are used, such as those used in this paper. This is because Wigner functions of separable states are themselves separable, $i$. e. not written as a products of functions of different degrees of freedom. Thus if we plot the Wigner function in such a way that any correlation between the phase space of one subsystem can be seen to be correlated with another we may not only see the entanglement correlations but also get deeper insight into the nature of these correlations over the phase-space. 
This will allow for general study beyond the Tavis-Cummings model and should also provide a framework for verification and validation of important quantum states for information processing and sensing applications.

\section{Composite systems in phase space}

We can calculate the Wigner function 7,8 of a system of arbitrary components by taking the expectation value of a suitable displaced parity operator over all its possible configurations - the phase space 9 -12. The total displaced parity operator for the composite system simply comprises the tensor product of the displaced parity operators for each element of the system.

In the Tavis-Cummings model the displaced parity operator for the field mode is the usual text-book displaced spatial parity $\hat{\Pi}_{f}(q, p)$. Each atom will be represented by the generalized displaced spin parity $\hat{\Pi}_{i}\left(\theta_{i}, \phi_{i}\right)$. This leads to a total system parity - and the kernel needed to generate the system's informational complete representation in phase space - as

$$
\hat{\Pi}\left(q, p,\left\{\theta_{i}, \phi_{i}\right\}\right)=\hat{\Pi}_{f}(q, p) \otimes\left[\bigotimes_{i=0}^{N} \hat{\Pi}_{i}\left(\theta_{i}, \phi_{i}\right)\right] .
$$

Specifically, the Wigner function will be

$$
W\left(q, p,\left\{\theta_{i}, \phi_{i}\right\}\right)=\operatorname{Tr}\left[\hat{\rho} \hat{\Pi}\left(q, p,\left\{\theta_{i}, \phi_{i}\right\}\right)\right] .
$$

To calculate the Wigner functions, all we need now is the method to determine each displaced parity.

For the field mode, the displaced parity operator $\hat{\Pi}_{f}(q, p)$ is constructed from the spatial parity

$$
\hat{\Pi}_{f}=\mathrm{e}^{\mathrm{i} \pi \hat{a}^{\dagger} \hat{a}}
$$

which is the operator that takes a coherent state $|\alpha\rangle$ to $|-\alpha\rangle$. We then displace this parity operator with the field displacement operator

$$
\hat{D}(q, p)=\exp (\mathrm{i}[p \hat{q}-q \hat{p}] / \hbar)
$$

where the displacement operator displaces a state in phase space. The coherent state $|\alpha\rangle$ is generated from the vacuum state $|0\rangle$, where $|\alpha\rangle=\hat{D}(\sqrt{2} \operatorname{Re}(\alpha), \sqrt{2} \operatorname{Im}(\alpha))|0\rangle$. The displaced parity operator for the Wigner function of the field mode is then defined in terms of these operators, where

$$
\hat{\Pi}_{f}(q, p)=\hat{D}(q, p) \hat{\Pi}_{f} \hat{D}^{\dagger}(q, p)
$$

Examples of the Wigner function for different field-mode states are given in Figs. 1 (a) - (d). Figs. 1 (a) and (b) show two coherent states, displaced in opposite directions from the origin, where $\alpha=3$. Fig. 1 (c) then shows the superposition of these two coherent states, resulting in the Schrödinger cat state in the field mode. We then show a statistical mixture of these two states in Fig. 1 (d). Comparing the pure, superposition state in Fig. 1 (c) with the statistical mixture in Fig. 11(d), we can see how the quantum correlations present as interference fringes between the two coherent states. 


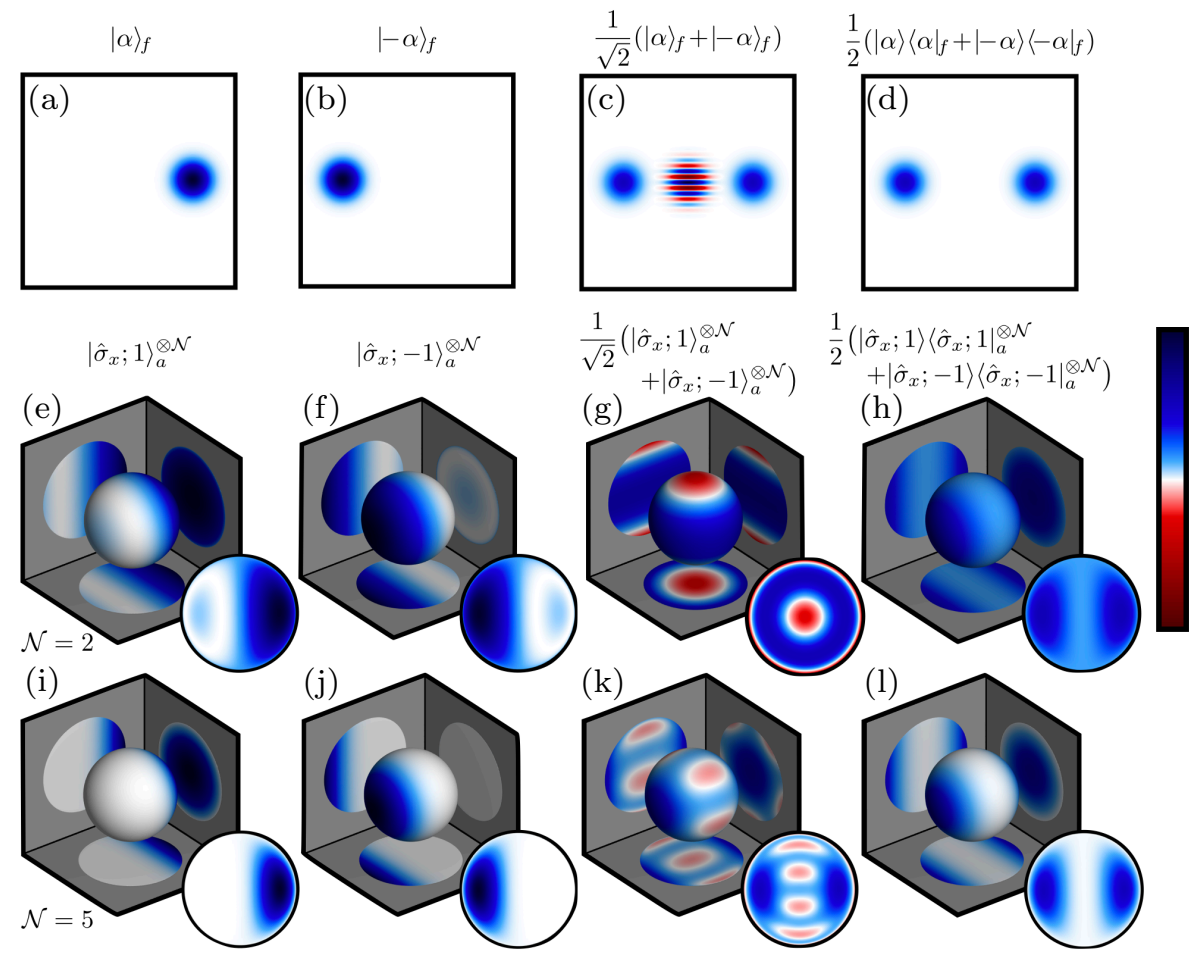

Fig. 1 Examples of the Wigner functions for coherent states in a field mode and multi-qubit states, where the eigenstates of $\hat{\sigma}_{x}$ are considered. Here, (a)-(d) show examples of coherent states in a field mode. Directly below are the two-qubit examples in (e)-(h). In the last row, (i)-(l), examples of the corresponding five-qubit states are shown. In the first two columns we consider simple coherent states for the respective systems. We then consider the superposition of these coherent states in the third column, (c), (g) and (k). This is followed by the statistical mixture of the coherent states in (d), (h) and (l). This figure is taken with permission from Ref. 13 .

The discrete analog of a coherent state, the spin coherent state, is defined similarly to a displaced vacuum state. Except now this displacement is a rotation over a spherical phase space. Such a rotation is defined by the Euler angles

$$
\hat{U}(\theta, \phi, \Phi)=\exp \left(\mathrm{i} \hat{\sigma}_{z} \phi\right) \exp \left(\mathrm{i} \hat{\sigma}_{y} \theta\right) \exp \left(\mathrm{i} \hat{\sigma}_{z} \Phi\right)
$$

A spin coherent state can then be generated by displacing $|\uparrow\rangle$, where $|\theta, \phi\rangle=$ $\hat{U}(\theta, \phi, \Phi)|\uparrow\rangle$ and $\hat{\sigma}_{z}|\uparrow\rangle=|\uparrow\rangle$. For multi-qubit states, the spin coherent state is the symmetric rotation of an initial tensor-product state $|\uparrow\rangle^{\otimes N}$. For a symmetric rotation, we then need to take the $N$-fold tensor product of $\hat{U}(\theta, \phi, \Phi)$, where the Euler angles are equal in each space. This results in the $N$-qubit spin coherent state $|\theta, \phi, \Phi\rangle_{N}=\hat{U}(\theta, \phi, \Phi)^{\otimes N}|\uparrow\rangle^{\otimes N}$.

To generate the displaced parity operator for the Wigner function, we then need to introduce the generalized parity operator for a single qubit

$$
\hat{\pi}=\frac{1}{2}\left(\mathbb{1}+\sqrt{3} \sigma_{z}\right),
$$


so that

$$
\hat{\Pi}_{i}\left(\theta_{i}, \phi_{i}\right)=\hat{U}_{i}\left(\theta_{i}, \phi_{i}, \Phi_{i}\right) \hat{\pi} \hat{U}_{i}^{\dagger}\left(\theta_{i}, \phi_{i}, \Phi_{i}\right),
$$

for Euler angles $\theta_{i}$ and $\phi_{i}$, where the index $i$ denotes that this is for the $i^{\text {th }}$ qubit. Note that the $\Phi_{i}$ degree of freedom cancels out as it commutes over the parity operator.

For a two-qubit state, we can then generate the kernel

$$
\hat{\Pi}\left(\theta_{1}, \phi_{1}, \theta_{2}, \phi_{2}\right)=\hat{U}_{1}\left(\theta_{1}, \phi_{1}, \Phi_{1}\right) \hat{\pi} \hat{U}_{1}^{\dagger}\left(\theta_{1}, \phi_{1}, \Phi_{1}\right) \otimes \hat{U}_{2}\left(\theta_{2}, \phi_{2}, \Phi_{2}\right) \hat{\pi} \hat{U}_{2}^{\dagger}\left(\theta_{2}, \phi_{2}, \Phi_{2}\right),
$$

this alone gives a function with four degrees of freedom. It is then necessary to reduce this in order to generate a visualization of the phase space. As we are considering symmetric states, we can then take the symmetric approach with the displaced parity operator; where, in analogy to the spin coherent state construction, we take what is known as the equal-angle slice. $i$. e. $\theta_{1}=\theta_{2}=\theta$ and $\phi_{1}=\phi_{2}=\phi$.

Examples of the two-qubit Wigner function plotted with the equal-angle slice are shown in Figs. 1 (e) - (h). We have also shown similar states for five qubits in Figs. 1 (i) - (l). Each distribution has been plotted on a sphere as well as projected onto a unit disc with the Lambert azimuthal area-preserving map [14. We have included both as the spherical presentation follows intuition much better, the benefit of the area-preserving map is that it allows us to see the full surface of the sphere while fully preserving the area - an important metric when considering probability distributions. As such, this representation will be preferred later on when considering the definition of an informationally complete Wigner function to visualize correlations in coupled atom-field systems.

Figs. 1 (e) and (f) show two spin coherent states, both on orthogonal sides of the sphere. We can see the Gaussian-like behaviour of these states on the spheres. The behaviour becomes clearer as we consider more qubits, where in Figs. 1 (i) and $(\mathrm{j})$ there are clear Gaussians, one on each side of the sphere.

Figs. 1 (g) and (k) then show the superpositions of these two spin coherent states for two and five qubits respectively, resulting in two- and five-qubit GHZ states. Like in the case for the field mode in Fig. 1 (c), this superposition results in oscillations in the Wigner function between the two coherent states. These oscillations fluctuate between positive and negative values, where the number of oscillations corresponds to the number of qubits in the GHZ state. Also like the field mode in Fig. 11(d), when we take the statistical mixture of the two coherent states, the oscillations disappear, and we're left with two coherent states, one on each side of the sphere.

For these state, we can see that the equal-angle slice of the Wigner function presents enough information to get a good visualization of the quantum correlations that manifest in multi-qubit states. However, this equal-angle approach is not possible when considering atoms coupled to a field mode. A method to visualize such states has been considered in Refs. [11, 15]. This method was used to visualize atomic orbitals in Ref. [15] and for considering the Jaynes-Cummings model in Ref. [11.

In order to visualize states with heterogeneous degrees of freedom we begin with the the phase-space distribution of the field mode. We then discretize this phase space into a grid. At each point we then plot the full Wigner function for 

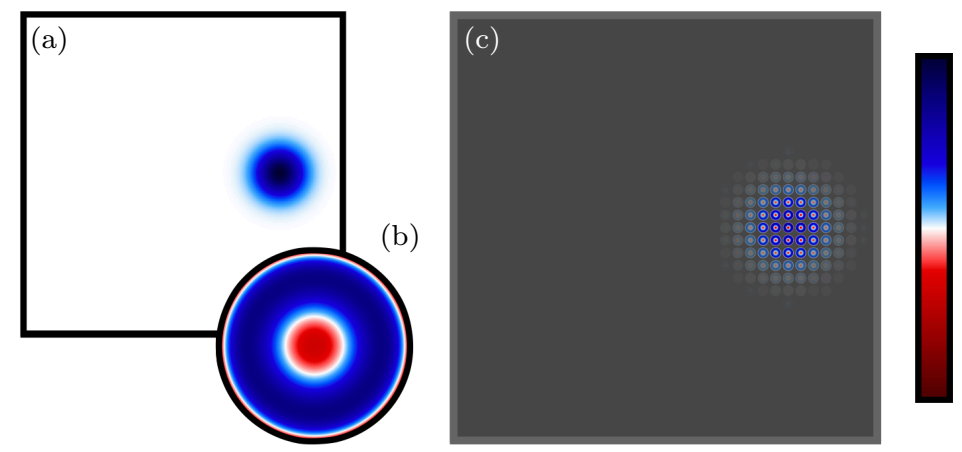

Fig. 2 Example of the full Wigner function for two atoms coupled to a field mode, showing the state $|\alpha\rangle(|\uparrow \downarrow\rangle+|\downarrow \uparrow\rangle) / \sqrt{2}$. This is also the initial state for the two-qubit Tavis-Cummings model. The reduced Wigner functions for the field mode and the atom are shown in (a) and (b) respectively. Our visulization of the full Wigner function is shown in (c). In order to maximise the available visual information at each point in phase space the opacity is set by the largest value of the Wigner function at this point in $(q, p)$ and we then plot the angular dependence as a small disc Lambert equal area autumnal plot at that position. In all future plots we sill simply refer to this kind of plot as the visualisation of the full Wigner function in order to avoid repetition. The colour bar goes between \pm 2 in $(\mathrm{a}), \pm(1+\sqrt{3} / 2)$ in (b), and $\pm(2+\sqrt{3})$ in (c). This figure is taken with permission from Ref. 13].

those set values of $q$ and $p$. Since we're dealing with two- and five- qubit states, the equal-angle slice is taken for the remaining degrees of freedom.

At every set value of $q$ and $p$ we then plot the remaining degrees of freedom. We then set the value of the opacity at that point in phase space proportional to the maximum absolute value over $\theta$ and $\phi$ at that point in position and momentum space.

As an example, see Fig. 2, where we show this method of visualizing the the coherent state from Fig. 1 (a) coupled to the GHZ state from Fig. 1 (g). These are shown as reduced Wigner functions in Fig. 2 (a) and (b) respectively. The full Wigner function is shown in Fig. 2 (c). Since there is no entanglement between the field mode and the two-qubit state, the Wigner function is then simply a replication of the reduced atomic state at every discrete point in position and momentum phase space. The opacity is then set by the coherent state in the field mode, which acts like an envelope over atomic states. When there are correlations between the degrees of freedom, this is no longer the case. Examples of these nonlocal correlations will be considered when we look at the Wigner function during the Tavis-Cummings evolution, which we will now discuss.

Note that much of the text and results that follow are taken, edited and adapted from Chapter 5 of RR's $\mathrm{PhD}$ thesis [13]. It has been extracted and reordered here as we believe that doing so provides an excerpt that is valuable as a stand alone piece. We note that we have also added the five-qubit Tavis-Cummings model with a coherent state as an initial state in the field mode in Section 4.2 that wasn't included in Ref. 13 . 


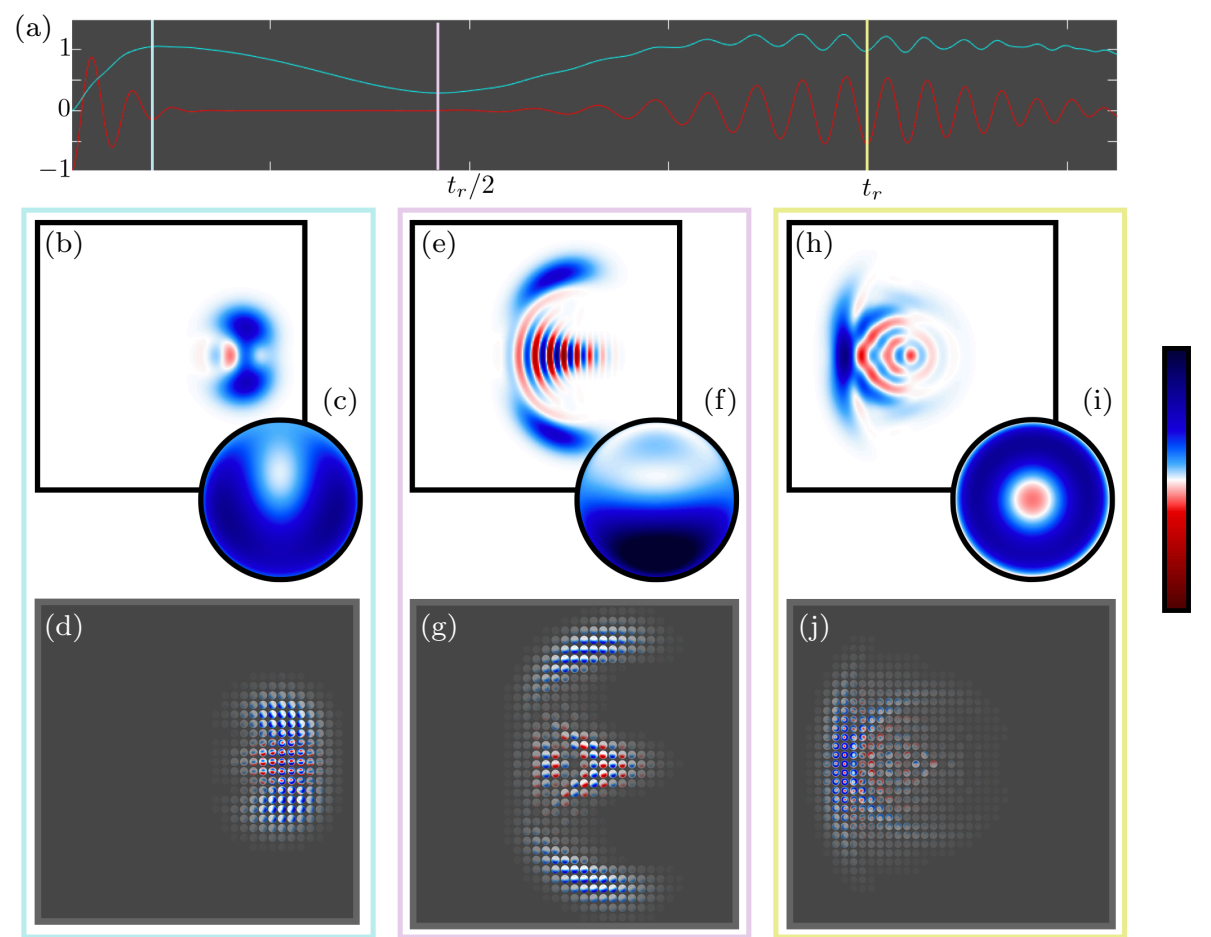

Fig. 3 The Wigner functions for three points in the evolution of the Tavis-Cummings model with initial state $|\alpha\rangle_{f}\left(|\uparrow \downarrow\rangle_{a}+|\downarrow \uparrow\rangle_{a}\right) / \sqrt{2}$. The graph in (a) shows the von Neumann entropy of the two qubits in cyan and the qubit inversion $\left(\left\langle\hat{\sigma}_{z}^{(1)}\right\rangle\right)$ in red throughout the entire evolution. The three points are marked out on this graph by vertical coloured lines. The Wigner functions for the first point are shown in (b), (c), and (d), showing an entanglement between the field mode and the qubits. The second point is shown in (e), (f), and (g), where the atom and field mode have decoupled, where the atomic Schrödinger cat state has been swapped into the field mode, showing a spin coherent state in the atomic state and a Schrödinger cat state in the field mode. The last point is a at the point of the revival of the Rabi oscillations at $t=t_{r}$, where the Wigner functions are shown in (h), (i), and (j). The colour bar values go between \pm 2 for the reduced Wigner functions for the field mode in (b), (e), and $(h), \pm(1+\sqrt{3} / 2)$ for the reduced atomic Wigner functions in (c), (f), and (i), and between $\pm(2+\sqrt{3})$ for the visualisation of the full Wigner function in $(\mathrm{d}),(\mathrm{g})$, and $(\mathrm{j})$. This figure is taken with permission from Ref. [13].

\section{Two-atom Tavis-Cummings interaction}

We will now consider a two-qubit example of the Hamiltonian in Eq. (1), starting with the initial state

$$
\frac{1}{\sqrt{2}}|\alpha\rangle_{f}(|\uparrow \downarrow\rangle+|\downarrow \uparrow\rangle)
$$

where $\alpha=3$, the Wigner function for this state has already been presented in Fig. 2.

Further points in the evolution of the two-qubit Tavis-Cummings model can be found in Fig. 3. Here we show snapshots at three points of interest during the evolution. In Fig. 3 (a) we show the von Neumann entropy over time in cyan, 
where

$$
S(\rho)=-\operatorname{Tr}\left[\rho_{a} \ln \rho_{a}\right],
$$

where $\rho_{a}=\operatorname{Tr}_{f}[\rho]$, is the reduced density matrix of the two-qubit state, tracing out the field mode. Note that we consider the entropy of the atom pair rather than the atoms individually, this in order to calculate the coupling between the atoms as a pair with the field mode. Also in In Fig. 3 (a), the atomic inversion is shown in red, where we take $\left\langle\hat{\sigma}_{z}^{(1)}\right\rangle$ of the state through time.

The first point of interest is the first local maximum of entropy, indicated in Fig. 3 (a) by the vertical blue line. The Wigner functions for the state at this point in the evolution are shown in the blue box in Figs. 3 (b) - (d). This shows the emergence of a Schrödinger cat state in the field mode. The reduced two-qubit state is quite clearly heavily mixed, which is to be expected as this is a point of high entropy. Turning our attention to the Wigner function, we again get the overall shape of a Schrödinger cat state in the field mode, where the atomic states in each of the two cats are pointing in almost orthogonal directions. The top of the Schrödinger cat state has spin coherent state pointing towards to left and the bottom cat is made of spin coherent states orientated to the right.

The quantum correlations between the atoms and the field mode becomes apparent when considering the area between the two cats. The quantum correlations manifest here with high Wigner-function values, where in the reduced state this area has much lower values. In fact, the characteristic feature of entanglement here is that the atomic states here are traceless - the overall positive value is equal to the overall negative value. A feature of quantum correlation in informationally complete Wigner functions that was noted in Ref. [11].

The second point of interest is shown in Fig. 3 (a) by the vertical pink line, the corresponding Wigner functions are shown in the pink box Figs. 3 (e) - (g). At this point in the evolution, the field and the atoms are decoupling from the entanglement generated in the previous state. This can be seen in the entropy as it reaches a local minimum, close to the inital value of 0 . This is where the information from the qubit has transferred to the field mode, swapping the atomic Schrödinger cat state to a Schrödinger cat state in the field mode. The two-atom state is in an almost-pure spin coherent state at every point in phase space, where the Wigner function for the field mode shows the definite signature of a Schrödinger cat state. The fact that the Wigner function is almost a direct product of the two reduced Wigner functions is demonstrative of the extent that the two systems have decoupled.

The third point is during the revival phase, given by the vertical yellow line in Fig. 3 (a). At this point, the cat has swapped back to the qubit, resulting in the atomic Schrödinger cat state being returned, as can be seen in the Wigner functions for this state in Figs. 3 (e) - (g). The field mode, in Fig. 3 (h), however isn't the initial coherent state. There is an identifiable concentration of quasiprobabilities on the left-hand side, forming a somewhat squeezed coherent state. The correlations in the middle of the field mode resemble a sort of noisy number state. Similar patterns emerge in the Wigner function in Fig. $3(\mathrm{j})$, where there are atomic cat states in the main coherent lump on the left-hand side, with some residual entanglement in the part that resembles a number state. 


\section{Five-atom Tavis-Cummings interaction}

Demonstrations of how quantum information can be shared between discrete variable system (atoms) and continuous variable systems (a field mode) have so far been given in this section. This has so far been shown for just two-qubit models. Where it was shown how a Schödinger cat state can be transferred to the field mode from a two-atom state. For the two-qubit Tavis-Cummings model, this was achieved by having the field mode initially in a coherent state $|\alpha\rangle$, at $\alpha=3$. This was done in order for the cat to faithfully swap from the two-atom state into the field mode.

Now, we will consider what happens in the five-qubit Tavis-Cummings model. We first investigate how quantum information can be shared from an initial atomic Schödinger cat state to a field mode in the vacuum state in Section 4.1, to see how this compares to both the two-qubit model considered earlier in this Chapter. We will then go on to see how starting with an initial vacuum state compares to starting the same evolution with an initial coherent state in the field mode in Section 4.2. We note that the results Section 4.1 are taken from RR's thesis [13. The following results in Section 4.2 are new results that were not included in Ref. 13].

\subsection{Vacuum state}

Here we consider the initial hybrid state

$$
\frac{1}{\sqrt{2}}|0\rangle_{f}\left(\left|\hat{\sigma}_{x} ; 1\right\rangle_{a}^{\otimes N}+\left|\hat{\sigma}_{x} ;-1\right\rangle_{a}^{\otimes N}\right)
$$

Like the Jaynes-Cummings model with a vacuum initial state - see Ref. 11] to see a demonstration of this in phase space - information is transferred back and forth between the atom and the field modes with each cycle of the Rabi oscillations. However, like the coherent state case, these Rabi oscillations collapse and revive over time, meaning that there is not a perfect transfer of quantum information from the initial atomic Schrödinger cat state the the field mode. This will give an opportunity to consider the full Wigner function as a tool for understanding impurities in a system, allowing an understanding of increased entropy between the systems.

Figure 4 shows the evolution of the initial state in Eq. 13 with the fiveatom Tavis-Cummings model Hamiltonian. Here, two periods of the Rabi cycle are shown, starting with the initial state. The next point is then after one cycle of the Rabi oscillations where the Schrödinger cat state has first been transferred to the field mode. Following is after the second cycle of the Rabi oscillations, where the Schrödinger cat state has returned to the atoms, regenerating a five-qubit atomic Schrödinger cat state.

The initial state is shown in Figs. 4 (a) - (c), where, as expected, there is a vacuum state in the field mode and a five-atom Schrödinger cat state. This produces the full Wigner function of an envelope of a coherent state where there is a GHZ state at every point in phase space. Note here that the colour bar has been slightly adapted from earlier figures, this is in order to highlight the oscillations 

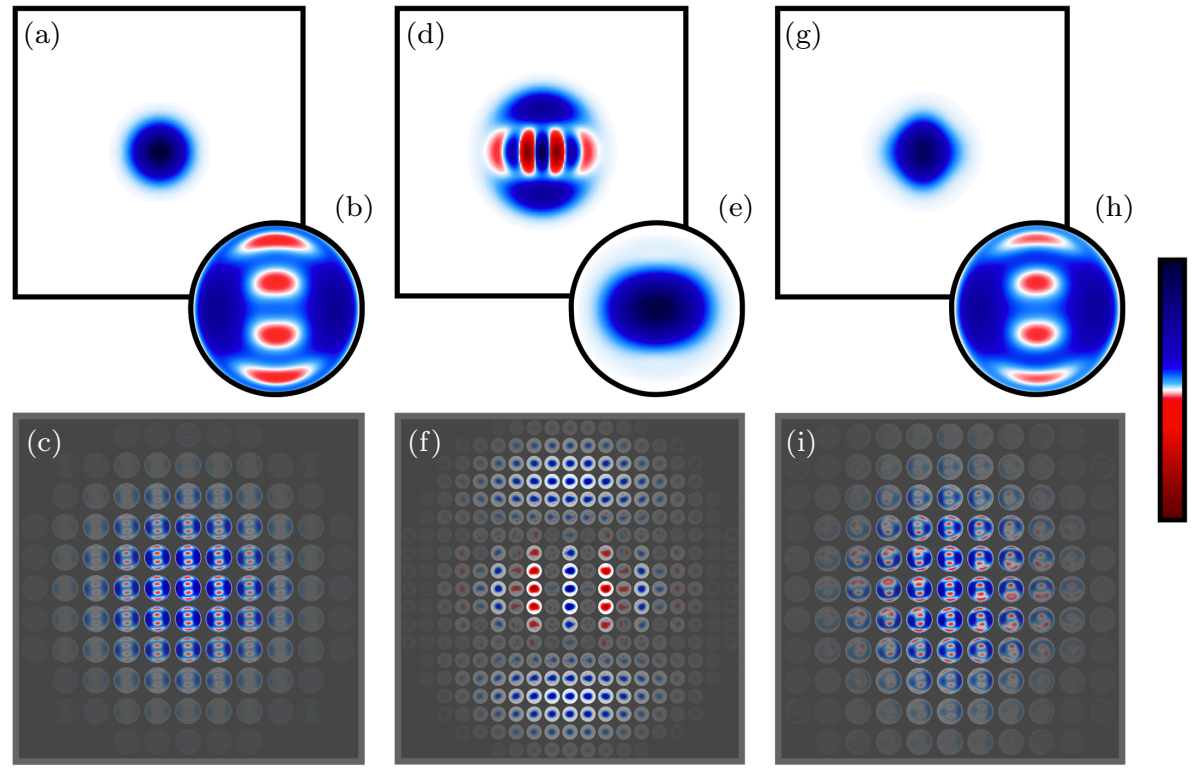

Fig. 4 Points in the five-qubit Tavis-Cummings evolution with an initial state of $|\alpha\rangle_{f}\left(\left|\hat{\sigma}_{x} ; 1\right\rangle_{a}^{\otimes 5}+\left|\hat{\sigma}_{x} ;-1\right\rangle_{a}^{\otimes 5}\right) / \sqrt{2}$. The top row, (a), (d) and (g), show the reduced Wigner functions for the field mode, with the reduced atomic Wigner functions inset in (b), (e) and (h). The visualisation of the full Wigner function for these states are shown in the bottom row, in (c), (f) and (i) respectively. Note that for all the atomic Wigner functions, the equal-angle slice has been taken. This figure shows the evolution from the initial state in (a)-(c) to where the quantum information transfers to the field mode in the form of a Schrödinger cat state in (d)-(f) and then returns to the atomic state in (g)-(i), albeit with some noise from an imperfect transfer of quantum information. Note here how there is a difference in the colourbar from earlier figures, this was done in order to highlight the oscillations in the atomic Schrödinger cat states. The maximum and minimum values are \pm 2 for the Wigner function for the field mode, $\pm((1+\sqrt{3}) / 2)^{5}$ for the five-qubit Wigner function, and $\pm 2((1+\sqrt{3}) / 2)^{5}$ for the visualisation of the full Wigner function. This figure is taken with permission from Ref. 13].

in the GHZ state. The range of position and momentum has also been decreased, in order to more clearly see the finer detail in the atomic states.

Figures 4(d) - (f) show the point at which the Schrödinger cat state has been swapped to the field mode. In Fig. 4 (d) the reduced Wigner function for the field mode shows the Schrödinger cat state, while Fig. 4. (e) shows the reduced Wigner function for the atoms, in the equal-angle slice. Since the quantum information has left, the atomic state now resembles an atomic coherent state. Since the transfer in this model is not a perfect state transfer, like in the Jaynes-Cummings model example, the atomic coherent state appears somewhat squeezed. Inspection of the visualisation of the full Wigner function in Fig. 4 (f) reveals why this is the case. At some points in phase space, the coherent state has more faithfully transferred to the atomic state, however at most points residual entanglement can be seen in the full state.

Next in Figs. 4 (g) - (i) is the point where the Schrödinger cat state returns to the atom. Like in Figs. 4 (d) - (f), this is not a perfect transfer of quantum informa- 

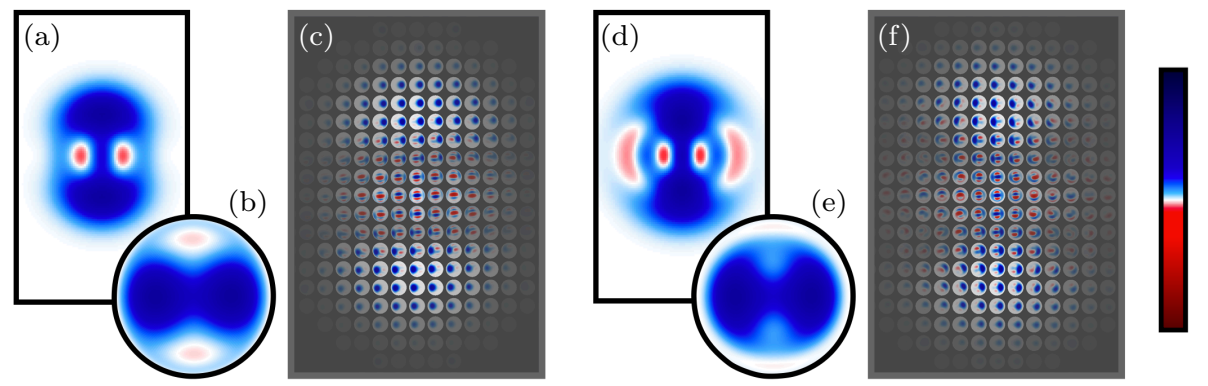

Fig. 5 The Wigner function for two points in the five-qubit Tavis-Cumming model. The points chosen here are between the points in the evolution shown in Fig. 4 where (a)-(c) is between the first two points of Fig. 4 and (d)-(f) are between the second and third points in Fig. 4 Fig. 4 showed the points at which the information had been transferred between the systems, are points where the information is halfway through being transferred, creating entangled hybrid Schrödinger cat states. The colour bar values go between \pm 2 for the reduced Wigner functions for the field mode in (b) and $(e), \pm((1+\sqrt{3}) / 2)^{5}$ for the reduced atomic Wigner functions in (b) and (e), and between $\pm 2((1+\sqrt{3}) / 2)^{5}$ for the visualisation of the full Wigner function in (c), and (f).

tion as impurities can be seen in both the reduced Wigner functions in Figs. 4 (g) and $(\mathrm{h})$ for the field mode and the atoms respectively. The main difference can be seen in the atomic Wigner function in Fig. 4 (h) where the oscillations in the GHZ state are lower in amplitude. However, only when looking at the visualisation of the full Wigner function in Fig. 4 (i) it is clear why there is this decrease in amplitude. Here, it can be seen at the origin of the field mode, a strongly formed GHZ state is present, however as you get further away from the centre, imperfections appear in the full quantum state.

From these three points in the initial Rabi oscillations for the Tavis-Cummings model, it can be seen by considering the Wigner function how information transfers from one system to the other. Further, it shows how the transfer has not been perfect, displaying noise around the full phase space, indicating a non-zero entropy in the system.

However, a high-entropy system may be what is desired in this information transfer, as in the Jaynes-Cummings model where, at the points between the transfer of the information, there were highly entangled states generated between the atom and the field mode. This is also the case in the five-atom Tavis-Cummings model.

These highly entangled midpoints are shown in Fig. 5 , where Figs. 5 (a) - (c) show a point between the first two points in Fig. 4 and Figs. 5 (d) - (f) show a point between the second and third points in Fig. 4. At both points, it can be seen in the reduced Wigner functions that there is a drop in quantum correlations for what would be expected for a Schrödinger cat state in either systems. Further the reduced Wigner function for the atomic states in Figs. 5(b) and (e) shows the two atomic coherent states moving closer together in phase space.

In the visualisation of the full Wigner functions in Figs. 5 (c) and (f) the two atomic coherent states can been seen in each of the field-mode Schrödinger cats, where they are pointing in different directions in each of the cats, at the top and the bottom of the figures, in the field mode. Between the two cats, in 
the centre of Figs. 5 (c) and (f), the emergence of quantum correlations from the entanglement between the different systems can be seen from the manifestation of traceless states.

It can further be seen that the first entangled state in Fig. 5 (c) is much cleaner that the state in Fig. 5 (f) later on in the evolution. The atomic states in Fig. 5 (c) are quite pure with little noise when far away from the correlations in the centre. However, the atomic states in Fig. 5 (f) are far more noisy, where the purest atomic coherent state exhibits some level of quantum interference, shown by the increased manifestation of negative quasi-probabilities.

\subsection{Coherent state}

The collapse and revival of entanglement in the Tavis-Cummings model has a much richer variety of behaviour than the simple Jaynes-Cumming model. It has been shown, for example that attractor states exist and that there is an associated basin of attraction for these states [3]. For initial conditions within this basin of attraction it will evolve to an attractor state at a specific times where all entanglement has, to all intents and purposes, left the system. Unlike in the Jaynes-Cummings model, such behaviour only happens for those initial states in the basin of attraction. For other initial states there can be very different and interesting behaviour. The ability to visualize such entanglement dynamics in addition to the usual non-local correlation dynamics shown by Wigner functions is thus of great interest. Let us reconsider the same setup as in Fig. 4 , but this time we displace the initial state of the field according to

$$
\frac{1}{\sqrt{2}}|\alpha=3\rangle_{f}\left(\left|\hat{\sigma}_{x} ; 1\right\rangle_{a}^{\otimes N}+\left|\hat{\sigma}_{x} ;-1\right\rangle_{a}^{\otimes N}\right) .
$$

This is the same idea as explored in [4] but now we are able to produce the Wigner function for the entire system.

In Fig. 6 the top panel Fig. 6 (a) shows the atomic inversion and entanglement entropy between the field mode and the the set of qubits. In the panels below this, for the three time points indicated in Fig. 6 (a), are the Wigner function for the reduced density matrix of the field in Figs. 6(b),(e) and (h). Figs. 6](c),(f) and (i) are the reduced density matrix of the qubits, and Figs. 6 (d), (g) and (j) are our visualization of the system's full Wigner function, using the equal angle slice for the spin degrees of freedom.

Here, the reduced Wigner functions show similar information to that presented in 4]. In particular Figs. 6 (e) and (f) show that the Schrödinger cat state has swapped from the spins into the field and Figs. 6. (h) and (i) show Schrödinger cat state swap back to the field mode.

In Figs. 6 (d),(g) and (j) we get additional information. Specifically we can see that the relative orientations (as an indicator of entanglement correlations) of each of the spin plots in Fig. 6 (g) are less significant than those in Fig. 6 (d) and are even more pronounced in Fig. 6 (j). In this way we can see that the different levels of entanglement in each state is manifest in each plot. We note as a word of caution that this approach should not be be viewed as representing an entanglement monotone - it is not - and it is important to remember that we are only looking at a much reduced part of a 12 dimensional phase space. 


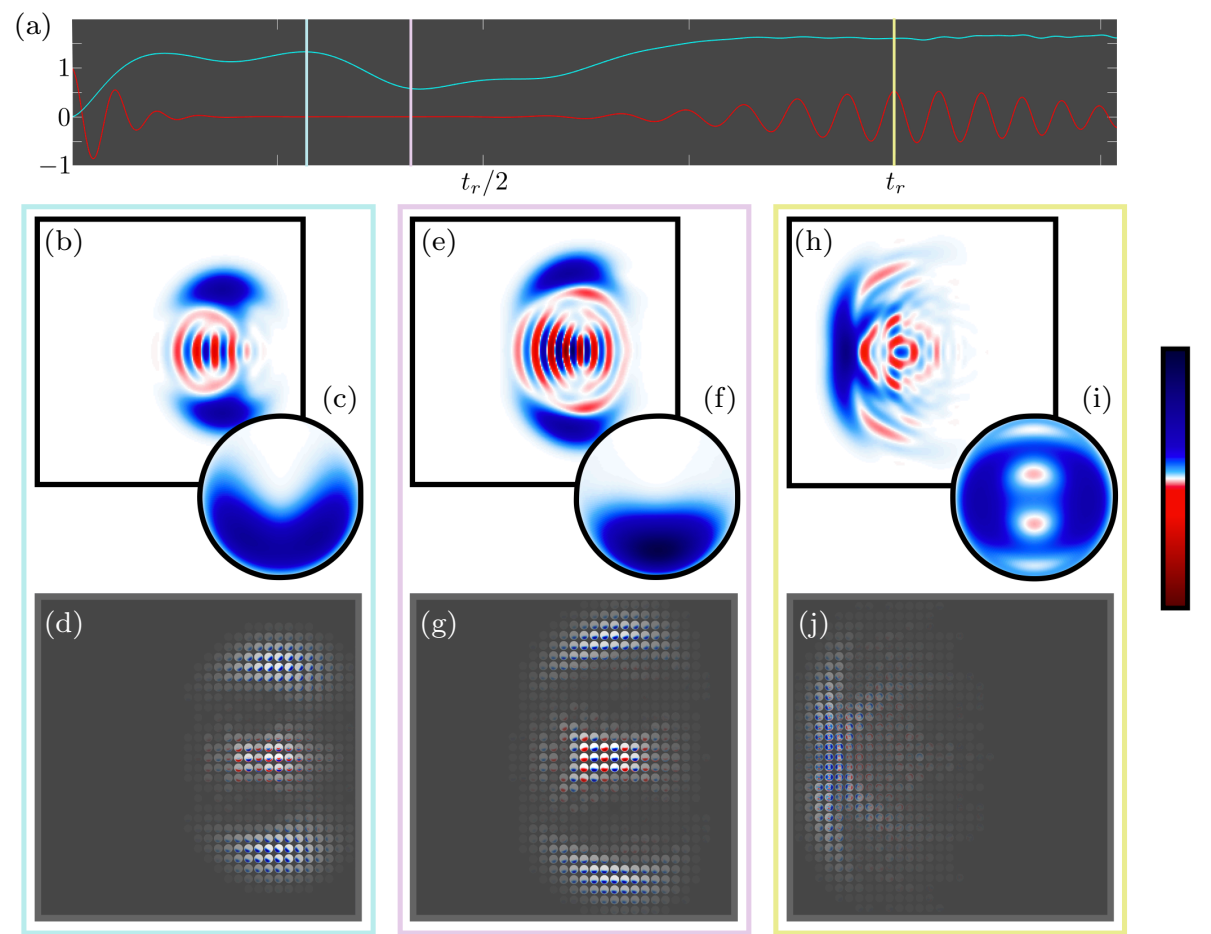

Fig. 6 Points of interest in the Tavis-Cummings model for a five-qubit state. Here, the initial is given in Eq. (14), which differs from the results in Fig. 5 as the initial vacuum state in the field mode is displaced. The initial state is not included here as it is visually similar to Fig. 4 just displaced in the field mode. The resulting in evolution afterwards has some differing features. Like in the two-qubit case we have chosen three points of interest, indicated by the vertical lines in (a). (a) also shows the von Neumann entropy of the reduced five-qubit state in cyan. The qubit inversion is then given in red. Like in the two-qubit case, the three points correspond to the initial maximal entropy point, where the field mode and atoms fully couple, generating an entangled Schrödinger cat state. This is followed by a decoupling of the two subsystems to a point of local minimum entropy, this is the point where the Schrödinger cat state has swapped to the field mode. We finally take the revival point where the atomic Schrödinger cat state returns to the five-qubit state.

In terms of additional information we can also see from $e . g$. (d) that if we make a joint $(q, p)$ measurement of the field state of this system to be in the top right segment we will know that the spins are in a state close to a spin coherent state with one orientation but if the results comes back in the lower right quadrant the ordination of the spin coherent state will be in the other orientation. Note that as the relevant ares of the phase space for the field mode needed to determine these different outcome are larger the a plank cell, which can also be determined from our visualizations, such joint measurement should be possible without violating the uncertainty principle. 


\section{Conclusions}

By taking a holistic approach to phase-space methods, rather than just analyzing the reduced Wigner functions, it is now possible to understand local and non-local correlations in quantum states. In this work we have demonstrated how our approach allows an intuitive visualisation of the process of swapping quantum information between two different systems. The understanding of this kind of process is useful, especially with the development of technologies such as quantum memory. Understanding how efficiently information can be swapped between one system to another will provide a way into understanding how efficient such technologies are, as the efficacy of quantum memory can only be as good as the information received from the state.

Our method does more than provide a reliable route to visualize, verify and validate hybrid and composite quantum systems. We have observed it also provides an mechanism for certifying quantum state preparation where $e . g$. making a joint measurement of the phase space of an ancillary system (in the model here - the field mode) certifies the state of the rest of the system (here the set of spins). We are confident that many more applications in sensing and validation of quantum states will arise as we further explore more and more complex systems in their entire phase-space.

Acknowledgements RPR acknowledges support from EPSRC grant number EP/N509516/1 and grant number EP/T001062/1 (EPSRC Hub in Quantum Computing and Simulation). All data generated or analysed during this study are included in this published article

\section{References}

1. E.T. Jaynes, F.W. Cummings, Proceedings of the IEEE 51(1), 89 (1963). DOI 10.1109/ PROC.1963.1664

2. M. Tavis, F.W. Cummings, Phys. Rev. 170, 379 (1968). DOI 10.1103/PhysRev.170.379. URL https://link.aps.org/doi/10.1103/PhysRev.170.379

3. C.E.A. Jarvis, D.A. Rodrigues, B.L. Györffy, T.P. Spiller, A.J. Short, J.F. Annett, New Journal of Physics 11(10), 103047 (2009). DOI 10.1088/1367-2630/11/10/103047. URL https://doi.org/10.1088/1367-2630/11/10/103047

4. M.J. Everitt, W.J. Munro, T.P. Spiller, Phys. Rev. A 85, 022113 (2012). DOI 10.1103/ PhysRevA.85.022113. URL http://link.aps.org/doi/10.1103/PhysRevA.85.022113

5. J.P. Dowling, G.S. Agarwal, W.P. Schleich, Phys. Rev. A 49(5), 4101 (1994). DOI 10.1103/PhysRevA.49.4101

6. R.H. Dicke, Phys. Rev. 93, 99 (1954). DOI 10.1103/PhysRev.93.99. URL http://link. aps.org/doi/10.1103/PhysRev.93.99

7. E.P. Wigner, Phys. Rev. 40, 749 (1932)

8. M. Hillery, R.F. O'Connell, M.O. Scully, E.P. Wigner, Phys. Rep. 106(3), 121 (1984)

9. T. Tilma, M.J. Everitt, J.H. Samson, W.J. Munro, K. Nemoto, Phys. Rev. Lett. 117, 180401 (2016). DOI 10.1103/PhysRevLett.117.180401. URL https://link.aps.org/doi/ 10.1103/PhysRevLett.117.180401

10. R.P. Rundle, P.W. Mills, T. Tilma, J.H. Samson, M.J. Everitt, Phys. Rev. A 96, 022117 (2017). DOI 10.1103/PhysRevA.96.022117. URL https://link.aps.org/doi/10.1103/ PhysRevA.96.022117

11. R.P. Rundle, B.I. Davies, V.M. Dwyer, T. Tilma, M.J. Everitt, J Phys Commun 4(2), 025002 (2020). DOI 10.1088/2399-6528/ab6fb6. URL https://doi.org/10.1088\% 2F2399-6528\%2Fab6fb6

12. R.P. Rundle, M.J. Everitt, Advanced Quantum Technologies p. 2100016. DOI https:// doi.org/10.1002/qute.202100016. URL https://onlinelibrary.wiley.com/doi/abs/10. 1002/qute.202100016 
13. R.P. Rundle, Quantum state visualization, verification and validation via phase space methods. Ph.D. thesis, Loughborough University (2020). DOI 10.26174/thesis.lboro. 11962620.v1. URL https://ethos.bl.uk/OrderDetails.do?uin=uk.bl.ethos.808068

14. J.H. Lambert, Beiträge zum Gebrauch der Mathematik und deren Anwendungen (Berlin, Verlag der Buchhandlung der Relschule, 1772)

15. B.I. Davies, R.P. Rundle, V.M. Dwyer, J.H. Samson, T. Tilma, M.J. Everitt, Phys. Rev. A 100, 042102 (2019). DOI 10.1103/PhysRevA.100.042102. URL https://link.aps.org/ doi/10.1103/PhysRevA.100.042102 